\title{
Brain repair: cell therapy in stroke
}

This article was published in the following Dove Press journal:

Stem Cells and Cloning:Advances and Applications

2I February 20I4

Number of times this article has been viewed

\section{Dheeraj Kalladka \\ Keith W Muir}

Institute of Neuroscience and Psychology, University of Glasgow, Southern General Hospital, Glasgow, United Kingdom
Correspondence: Dheeraj Kalladka Institute of Neuroscience and Psychology, University of Glasgow, Southern General Hospital, Glasgow, United Kingdom Tel +44 I4I 20 I 2 I 44

Fax +44 I4l 2012136

Email dheeraj.kalladka@glasgow.ac.uk
Abstract: Stroke affects one in every six people worldwide, and is the leading cause of adult disability. Some spontaneous recovery is usual but of limited extent, and the mechanisms of late recovery are not completely understood. Endogenous neurogenesis in humans is thought to contribute to repair, but its extent is unknown. Exogenous cell therapy is promising as a means of augmenting brain repair, with evidence in animal stroke models of cell migration, survival, and differentiation, enhanced endogenous angiogenesis and neurogenesis, immunomodulation, and the secretion of trophic factors by stem cells from a variety of sources, but the potential mechanisms of action are incompletely understood. In the animal models of stroke, both mesenchymal stem cells (MSCs) and neural stem cells (NSCs) improve functional recovery, and MSCs reduce the infarct volume when administered acutely, but the heterogeneity in the choice of assessment scales, publication bias, and the possible confounding effects of immunosuppressants make the comparison of effects across cell types difficult. The use of adult-derived cells avoids the ethical issues around embryonic cells but may have more restricted differentiation potential. The use of autologous cells avoids rejection risk, but the sources are restricted, and culture expansion may be necessary, delaying treatment. Allogeneic cells offer controlled cell numbers and immediate availability, which may have advantages for acute treatment. Early clinical trials of both NSCs and MSCs are ongoing, and clinical safety data are emerging from limited numbers of selected patients. Ongoing research to identify prognostic imaging markers may help to improve patient selection, and the novel imaging techniques may identify biomarkers of recovery and the mechanism of action for cell therapies.

Keywords: stroke, cerebrovascular disease, cell therapy, neurological disease

\section{Introduction}

Stroke is the most common cause of adult-acquired disability in the developed ${ }^{1}$ and developing world. ${ }^{2}$ With an aging population, the incidence and prevalence of stroke are predicted to rise. ${ }^{3}$ Stroke is an acute-onset clinical syndrome that develops following a vascular insult to the brain. Brain ischemia resulting from thromboembolism or less frequently, in situ thrombosis, constitutes $80 \%-85 \%$, and hemorrhage resulting from hypertension or vessel wall pathology constitutes $15 \%-20 \%$ of all strokes. Following vascular occlusion, a complex chain of events occurs at a molecular level, leading to irreversible tissue injury, including failure of energy synthesis, loss of transmembrane ionic gradients dependent on active transport, cell depolarization, and excitotoxicity due to the excess release of excitatory neurotransmitters. In the region with severely reduced blood flow (the ischemic 
core), these processes result in rapid cell necrosis affecting all the cellular elements (neurons, glia, and blood vessels). A region around the core (the ischemic penumbra) transiently maintains a collateral blood supply sufficient for cell viability. Restoring perfusion can salvage penumbral tissue, and timely recanalization is the most robust predictor of good clinical prognosis following ischemic stroke. ${ }^{4}$ Early thrombolysis with intravenous recombinant tissue plasminogen activator increases the likelihood of recanalization and a recovery to independence defined on scales of disability and handicap. ${ }^{5}$ Alternative reperfusion strategies have not yet shown benefit. Secondary processes following ischemic injury and cell necrosis include an inflammatory response, with the activation of microglia, infiltration of tissue by neutrophils and macrophages from the blood, and blood-brain barrier breakdown. Inflammatory mediators can act as chemoattractants for both the endogenous and exogenous cells involved in tissue repair. At the network level, regions of the brain that were previously connected to the infarcted area reorganize, at least in terms of the brain activation patterns seen on functional magnetic resonance imaging (fMRI). Rehabilitation exploits the combination of functional reorganization and adaptation after stroke. ${ }^{6}$

Immediately after stroke, several events, including edema, deafferentation, and inflammation, occur around the infarct, and some early functional recovery can be attributed to the resolution of edema and inflammation. However, this is usually limited, and other processes, including immunomodulation, angiogenesis, endogenous neurogenesis, and altered gene expression, may be involved in the longer-term recovery of function. The apparent translational failure of neuroprotective strategies ${ }^{7}$ that aim to interrupt or slow the injurious postischemic biochemical/molecular events may be attributed to various factors, including the heterogeneity of clinical stroke populations, ${ }^{8}$ inadequate sample sizes, and dose-limiting drug toxicities. However, recent critiques of the preclinical literature have suggested that the discrepancies between the preclinical and clinical studies are likely also to have arisen from publication bias, ${ }^{9}$ the limited replication of results, and experimental methodological flaws that inflated the estimates of effect size and led to the potential selection of inappropriate therapeutic candidates. ${ }^{10}$ A series of Stroke Therapy Academic Industry Roundtable (STAIR) ${ }^{11}$ meetings produced recommendations on the minimum standards for preclinical evidence that should, ideally, underpin the selection of drug candidates for clinical testing, as well as the clinical trial methods. An equivalent process, entitled Stem Cells as an Emerging Paradigm in Stroke (STEPS), has provided a forum for methodological discussions in the cell therapy field. ${ }^{12,13}$

\section{Overview of stem cell therapy in stroke}

Stem cells are undifferentiated cells that have the capacity to self-renew and differentiate into a range of tissues. Stroke therapy has distinct requirements compared with other neurological diseases, like Parkinson's disease or multiple sclerosis, since stroke is nonprogressive, involves a focal loss of tissue of all cell types, and is typically associated with a degree of endogenous recovery. Stem cell therapy is not, therefore, restricted to a paradigm of the replacement of a tissue, or a specific neuronal cell type (the focus in Parkinson's disease, for example), but potentially extends to effects on inflammation, immunomodulation, and the stimulation of endogenous recovery. Cell therapies probably act on multiple mechanisms in ischemic stroke, depending upon the timing and mode of administration; however, unlike neuroprotectant drugs, cell therapies have the advantage that they may be able to respond dynamically to an environment that varies both temporally and spatially after ischemia, rather than targeting a single pathway or mechanism of action. Interaction with the host environment appears to dictate the phenotypic properties of stem cell grafts. Stem cells come from various sources, and although they share some common properties, they also differ in many respects and behave differently in terms of their rate of differentiation, trophic factor secretion, and in their stimulation of endogenous processes when in a pathologic environment. No studies have compared the different cell types in the same experiment.

\section{Endogenous stem cells}

Until the middle of the 20th century, it was generally believed that neurogenesis in the mammalian nervous system was restricted to fetal development and that regeneration did not occur in the adult brain. In 1965, Altman and Das ${ }^{14}$ first reported postnatal neurogenesis in the rat brain, and by the late 20th century, there was evidence of similar endogenous neurogenesis in humans. ${ }^{15}$ In animals and humans, neuroblasts are known to be produced in the subventricular zone, ${ }^{16}$ subgranular zone of the hippocampal dentate gyrus, ${ }^{17}$ and, albeit controversially, in the newly discovered subcallosal zone that lies between the hippocampus and corpus callosum in rats. ${ }^{18}$ Increased neuroblast production following ischemic stroke has been observed in the rat subventricular zone, and cortical neuroblasts have been reported in both a rat stroke model $^{19}$ and in human brain biopsy specimens of penumbral 
tissue that were acquired for diagnostic purposes after stroke. ${ }^{20,21}$ Neuroblast production has also been stimulated experimentally by extrinsic growth factors, like hepatocyte growth factor, ${ }^{22}$ and specific molecules, such as statins ${ }^{23}$ and fluoxetine, ${ }^{24}$ but few of these neuroblasts appear able to migrate to the boundary of ischemic damage, ${ }^{25}$ calling into question their functional relevance - amplifying and sustaining this endogenous poststroke neurogenesis response and overcoming the low rate of cell survival may be relevant for functional gains. An improved understanding of the role of changes in the expression of the developmental genes and associated proteins that are observed along the ischemic border after stroke ${ }^{26}$ may also be important in developing cell or pharmacologic augmentation therapies that will capitalize on endogenous neuroregenerative capacity.

Olfactory ensheathing cells are a self-renewing population of cells that display the properties of both glia and Schwann cells and are found at the junction between the central and peripheral nervous systems. Their main properties have led them to be studied more in the context of spinal cord and nerve root injuries, but their neuroplastic effects have been tested in murine models of stroke and they have been found to promote neurite outgrowth. ${ }^{27}$ Few preclinical studies exist, and their clinical application remains unclear in stroke. ${ }^{28}$

\section{Exogenous stem cells}

The application of exogenous cell therapy in neurology began with neurodegenerative diseases, for which fetal ventral mesencephalic tissue was transplanted with the intention of replacement of a specific cell type, such as the dopaminergic neurons of the basal ganglia. ${ }^{29}$ Cell replacement for stroke requires the regeneration of multiple functionally specialized cell types, with differing ratios in different brain regions, but extends also to glial cells and blood vessels since the injury involves the entire neurovascular unit.

\section{Neural stem cells}

Whether neural stem cells (NSCs) should be defined by their tissue of origin or their capacity to generate neural tissue is not universally agreed. ${ }^{15}$ The following discussion considers the tissue of origin to define NSCs. Cells sourced from ectodermal tissue, such as the central nervous system (CNS), have restricted differentiation potential and can further be categorized into embryonic, ${ }^{30}$ fetal, ${ }^{31}$ or adult, ${ }^{30}$ by origin. The use of adult-derived cells does not share the ethical and practical concerns of the use of embryonic or fetal cells. Cells from adult murine brain have been harvested, expanded in culture, and reimplanted as an allogeneic source. Isolated cells can be induced to form neurospheres, which are then expanded in vitro before delivery via various routes, including stereotactic (ST) injection to the brain, and intravenous (IV), intra-arterial (IA) and intracerebroventricular (ICV) administration. The differentiation spectrum of NSCs is restricted to neurons, astrocytes, or oligodendrocytes and can be influenced by intrinsic factors, ${ }^{32}$ such as neuron-restrictive silencing factor, and extrinsic factors, such as experimental hypoxia $^{33}$ and epidermal growth factors. The transmission of infectious agents by culture media is a concern that can be addressed only incompletely by applying strict Good Manufacturing Practice standards. Human fetal brain cortex cells have been immortalized by the insertion of $c-M y c^{31}$ and $v-M y c^{34}$ transcription factor genes, in order to enhance cell survival or allow the regulation of cell replication (for example, where $c-M y c$ expression is under the regulatory control of a modified estrogen receptor). ${ }^{35}$ The majority of NSC experimental stroke studies have used ST $\mathrm{ST}^{31,36-38}$ intracerebral delivery, with implantation ranging from hours ${ }^{39}$ to 6 weeks $^{38}$ after stroke. Cell migration to ischemic regions has been reported following implantation by $\mathrm{ST}^{38}{ }^{38},{ }^{40}$ or $\mathrm{IA}^{41}$ routes. ST-implanted human NSCs have migrated up to $1.2 \mathrm{~mm}$ in the lesioned hemispheres compared with $0.2 \mathrm{~mm}$ in naïve rat brain. ${ }^{42}$ Whether more distant migration occurs is unclear. Cell survival varies and depends on the timing and mode of delivery. Following ST implantation, proximity to the lesion influences survival, ${ }^{38}$ while very few cells reach the brain following IV administration as they are filtered by the pulmonary vascular bed and sequestered in the spleen. ${ }^{43}$ Slightly greater cell survival in the CNS is seen after IA delivery. ${ }^{44}$ Although many cells die early after administration, bioluminescent human NSCs ST-implanted 7 days after middle cerebral arterial occlusion (MCAo) have been observed to survive beyond 2 months, with over $50 \%$ cell survival confirmed on histology. ${ }^{45}$ Surviving cells exhibit a wide spectrum of fates, ranging from $78 \%$ remaining in an immature state ${ }^{36}$ at week 5 , to unquantified numbers of differentiated neurons forming synapses with host cells. ${ }^{45}$ The expression of neuronal cell surface markers does not necessarily indicate functioning neuronal tissue, still less, useful integration, and the contribution of the surviving cells to an observed functional improvement is still unclear. A change in neurological or behavioral function has been the preferred outcome, rather than infarct volume, as NSC studies have mostly chosen to implant at subacute time points, when infarcts are well-established. A modified neurological severity score (NSS), which provides a composite score 
based on motor, sensory, reflex, and balance responses, has been used commonly in preclinical rodent studies to assess change, reporting significant improvements compared with sham controls, following NSC therapy. ${ }^{46,47}$ However, a wide range of behavioral tests has been employed. The reporting of results differs across laboratories, ${ }^{48}$ and the reproducibility of tests across observers and also across time has seldom been reported. Despite the lack of clarity regarding the mechanisms of action, NSCs are believed to alter white matter tissue structure, and a noninvasive method to measure this would be valuable. The effects of NSC treatment on white matter reorganization can be monitored by measuring water diffu$\operatorname{sion}^{49}$ using magnetic resonance imaging (MRI) with diffusion tensor imaging (DTI) sequences. Although DTI in small animals is compromised by the relatively lesser volume of white matter present in rodents compared with humans, there are also some advantages to use of DTI, including the ability to apply longer scan acquisition times and higher magnetic field strength. The white matter reorganization observed on histology was coincident with improved fractional anisotropy, and fiber tracking maps revealed similar orientation patterns to that seen on immunohistology. ${ }^{41}$

\section{Mesenchymal stem cells}

Since the first bone marrow-derived mesenchymal stem cells (MSCs), ${ }^{50}$ many other cell types with similar properties from various tissues, including bone marrow mononuclear cells, adipose-derived stem cells, umbilical cord blood cells (UCBCs), endothelial progenitor cells (EPCs), peripheral blood progenitor cells, cluster of differentiation (CD)34+ cells from placenta, periosteal stem cells, and amniotic fluid cells have all been proposed as potential alternatives. The relative ease of cell acquisition without ethical difficulties has fuelled interest in MSCs, but the specific characterization of MSCs has not been consistent over time, ${ }^{51,52}$ making study comparability difficult. In vitro cultures contain a mix of committed and noncommitted progenitors that can form, not only mesodermal, but under certain circumstances, also ectodermal cell types, like neurons, but it is unclear whether the MSCs differentiated along neuronal lines in culture will have the same properties as do NSCs. Human neuronal MSCs, which have the ability to differentiate into neuronal cells following transfection of the Notch intracellular domain, were ST-implanted 4 days after MCAo in gerbils and compared with human MSC. In the human neuronal MSC group, better cell survival and functional recovery were observed despite the absence of synaptic connection between the transplanted and recipient cerebral cells on fluorescence-in-situ-hybridization (FISH), suggesting that the neuronal differentiation did not contribute to the MSC beneficial effects. ${ }^{53}$ In experiments with MSCs derived from donor rats, ${ }^{54}$ mice, ${ }^{55}$ rabbit, ${ }^{56}$ (autologous or allogeneic), or humans ${ }^{57}$ (xenogeneic), cells have been transplanted by $\mathrm{IV},{ }^{57} \mathrm{IA},{ }^{58} \mathrm{ST},{ }^{59}$ or intracisternal ${ }^{58}$ routes into animals, from hours $^{57}$ to 1 month $^{60}$ after induction of stroke with either temporary or permanent MCAo. Homing of the transplanted MSCs appears to occur via a complex multistep process that includes interactions with the stromal cell-derived factor 1 (SDF-1) (also called C-X-C motif chemokine 12 [CXCL12]) chemokine receptor. ${ }^{61}$ Homing signals originate from within the active inflammatory zone in the injured tissue. MSC migration to specific sites has been observed in stroke studies, where they have been found to travel preferentially to the ischemic boundary, following IV $^{57}$ and ST delivery. ${ }^{62}$ Few cells have been shown to survive in the studies of xenogeneic cell implantation. With no immunosuppression, cell survival of up to 2 weeks has been reported on ST implantation, but the proportion of surviving cells has not been quantified ${ }^{63}$ and has qualitatively been described as being a small proportion only. Long-term cell engraftment has not been detected with IV administration on histology. ${ }^{64}$ In another study, out of $3 \times 10^{6} \mathrm{MSC}$ delivered IV, only $3 \%$ of administered cells expressed neuronal markers in vivo, ${ }^{65}$ further supporting the concept that tissue replacement is not likely to be a functionally relevant mechanism of action for this cell type. Trophic factors, such as brain-derived neurotrophic factor (BDNF), glial-derived neurotrophic factor (GDNF), vascular endothelial growth factor (VEGF), neurotrophin-3 (NT3), fibroblast growth factor (FGF), and thrombospondins, secreted by the $\mathrm{MSCs}^{66,67}$ in response to the local microenvironment may, along with their stimulation of neurogenesis, ${ }^{66}$ angiogenesis, ${ }^{68}$ and immunomodulation, ${ }^{69}$ underlie functional recovery. Astrocytes are known to maintain normal neuronal function, ${ }^{70}$ forming an important pathway for endogenous repair. ${ }^{71}$ Exogenous MSCs have been observed to influence astrocyte survival and astrocyte trophic factor gene expression after anaerobic insult, by upregulating several kinase pathways and protein functions. ${ }^{72}$ After ischemia, astrocytes form gliotic scar tissue, which may be helpful in limiting tissue inflammation but can impede axonal regeneration. IA-implanted MSCs have shown histological evidence of improved axon-myelin remodeling after stroke, ${ }^{73}$ but it is unknown whether this mechanism is relevant in other routes of MSC administration. MSCs naturally adopt different trophic factor expression dependent on the injured host neural tissue. ${ }^{74}$ Higher levels of BDNF, NT3, and VEGF 
have been detected at the ischemic boundary 14 days after ST human MSC transplantation in rat brains compared with controls that received saline. ${ }^{75}$ The expression of VEGF and FGF has been consistently high at the ischemic boundary, potentially driving endothelial cell proliferation and angiogenesis, and facilitating regional blood flow. ${ }^{76}$ In contrast with the NSC studies, infarct volume has been the preferred outcome measure for experimental MSC therapy, which has been predominantly administered in an acute or early subacute IV delivery paradigm, with significant reduction in infarct volumes and good correlation noted between histology and imaging measures. ${ }^{77}$ Significant improvements have also been reported in behavioral measures, which have included assessments of sensorimotor function, motor coordination, and placing deficits during locomotion (treadmill test), ${ }^{77}$ forelimb function and placing deficits (limb placement test) ${ }^{78}$ motor coordination and balance (rotarod test), ${ }^{75}$ and a composite of motor, sensory, reflex, and balance responses (NSS).$^{79}$ Other cell types including UCBCs, EPCs, adipose-derived stem cells, and hematopoietic progenitor cells (CD34+ cells), share some of the properties of bone marrow-derived MSCs and have been found to have similar effects in animal models. In animal experiments, UCBCs respond to ischemic region homing signals, migrate to the lesioned hemisphere following IV administration, and differentiate, as evidenced by immunohistochemical neuronal and astrocytic markers. ${ }^{80}$ Some MCAo rat studies have failed to detect IV-administered UCBC in the lesions despite improvement in spontaneous activity and behavioral motor tests, suggesting a trophic factor-mediated response. ${ }^{81,82}$ CD34+ cells form a significant component of UCBCs that have been enriched either from the umbilical cord, peripheral blood, or bone marrow, and administered separately. IV administration of CD34+ in MCAo models has shown ischemic border zone neovascularization that has in turn, stimulated endogenous neurogenesis. ${ }^{83}$ EPCs represent cells with varying cell expression markers, ${ }^{84}$ typically CD34+, $\mathrm{CD} 133+$, and kinase insert domain receptor (KDR+) (also known as vascular endothelial growth factor receptor-2), with an angiogenic mechanism of action and found to reduce infarct volumes in rat stroke models when administered IV a day after MCAo. ${ }^{85}$ Several Phase I and II MSC clinical trials are ongoing (Table 1).

\section{Embryonic stem cells}

Embryonic stem cells (ESCs) are derived from the first stages of embryonic development: the first human ESC lines were established in 1998 from the inner cell wall of the blastocyst stage ${ }^{86}$ Religious and moral objections have been raised to the medical use of embryonic material; however, it is not widely recognized that the ESCs used for medical research are generally obtained from in vitro fertilization programs. Media reports often fail to distinguish ESCs from other stem cell types, leading to public confusion. ESCs are pluripotent and able to differentiate into tissues of all three germ layers. Although at first glance this might appear advantageous, regulatory control over ESC differentiation may be necessary before therapeutic use, since ESCs tend to form teratomas when grafted, ${ }^{87}$ with the postischemic environment possibly promoting teratoma formation. ${ }^{88} \mathrm{ESC}$ studies in animal stroke models have been concerned with mechanistic aspects rather than functional efficacy, and report only isolation, neutralization, ${ }^{89}$ and the electrophysiological activity of differentiated neuronal cells. ${ }^{90}$ Undifferentiated ESCs grafted into rat brains have differentiated and integrated with host tissues in stroke models, ${ }^{91}$ showing improved functional outcomes on the cylinder test, which measures the spontaneous use of forelimbs. ${ }^{92}$

ESCs remain widely researched as a source for in vitro generation of neuronal cell lines for drug screening, mechanistic investigation, or therapeutic use. ESCs can be stimulated to differentiate into specific neuronal populations or glia, with appropriately timed use of growth and inhibitory factors in relevant media and culture conditions. ESCs have been preferentially differentiated to a glutamatergic neuronal phenotype of the auditory nerves, with a view to specific tissue regeneration of the auditory nerve. ${ }^{93}$ Similar preferentially differentiated cell cultures can be used for in vitro studies, to investigate several critical stroke-related molecular processes. Such studies provide tight experimental control despite limitations of their ability to investigate the role of cell interactions. The cellular effects of oxygen-glucose deprivation, hypothermia, oxidative stress, and excitotoxicity have been modeled with chosen degrees of injury, helping to improve our understanding of certain key pathological processes. ${ }^{94}$

\section{Induced pluripotent stem cells}

Nobel Laureate Shinya Yamanaka and his colleague Kazutoshi Takahashi first demonstrated that differentiated murine cells could be reprogrammed to an embryonic-like state, with cells having the morphology, growth properties, and cell surface markers of ESCs, calling them induced pluripotent stem cells (iPSC). Similar iPSCs were later derived from adult human somatic cells. ${ }^{95}$ The Yamanaka method involved the transfection of cells with four key nuclear transcription factors, under ESC culture conditions; $;{ }^{96}$ subsequent studies have 


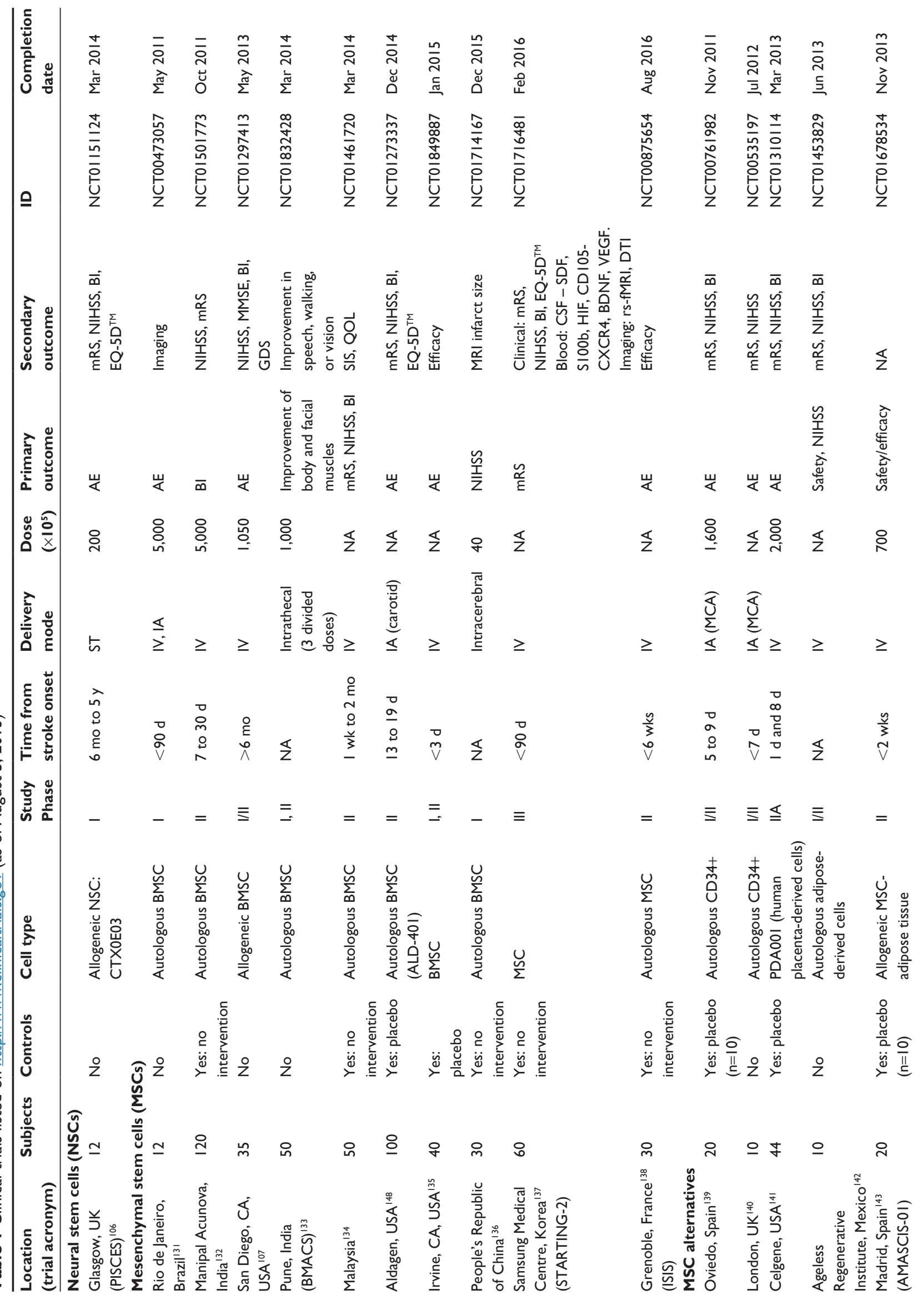




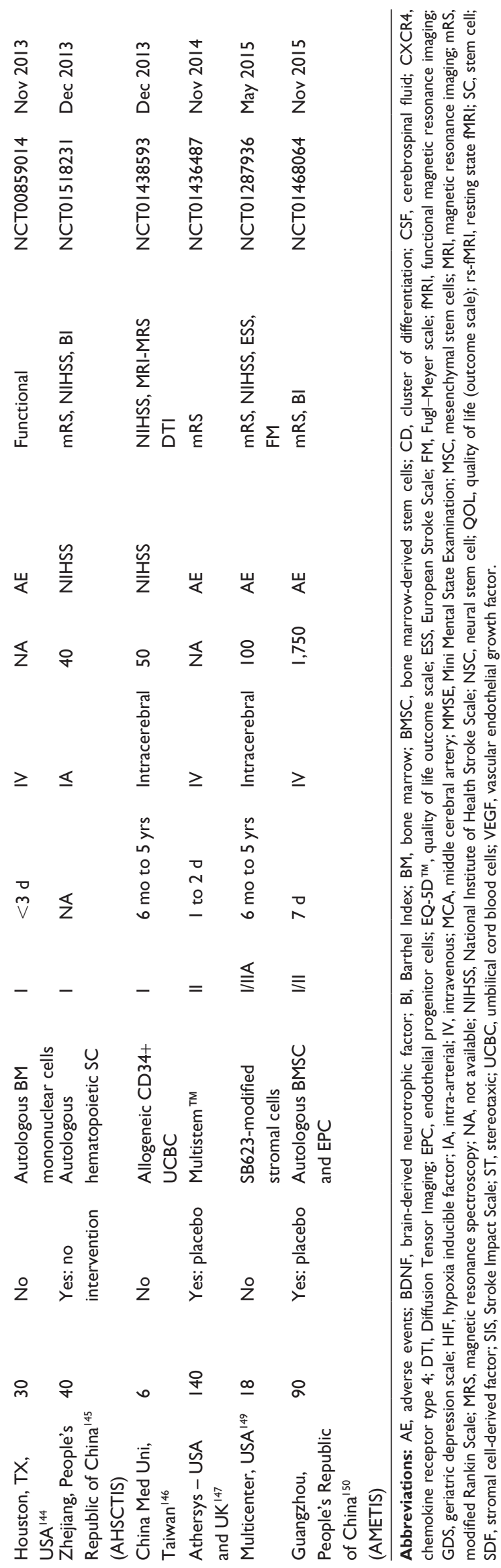

identified alternative methods. ${ }^{97}$ While superficially appealing as a means of obtaining ESC-like cells from adult tissue, the limited yield of these methods, the potential risks of clinical use of material obtained from viral transfection, and the multiple potentially oncogenic transcription factor genes, as well as (for stroke) the time required for culture expansion, all present significant clinical hurdles that are currently being investigated. ${ }^{98}$ iPSCs can potentially generate autologous patient-specific cells, avoiding the ethical, moral, and legal issues of ESCs but may share the tumorigenicity issues of ESCs. ${ }^{87}$ The intracerebral implantation of undifferentiated iPSCs in a rat MCAo model showed cell expansion to form large tridermal teratomas, with little behavioral improvement compared with controls, despite differentiated neuroblasts and mature neurons being seen in the ischemic lesion. ${ }^{99}$ As is the case for ESCs, partial in vitro differentiation may be necessary before therapeutic uses can be contemplated. A recent study that used human iPSC-derived long-term expandable neuroepithelial-like stem cells in a T cell deficient rat MCAo model with a 4-month observation period found no new tumors or transplant overgrowth, suggesting that predifferentiation of iPSCs and the generation of long-term self-renewing neural cell lines may offer an effective strategy for minimizing the risk for tumor formation. ${ }^{100}$ The reports of improvement in function, reduced infarct volume, and differentiated neuronal cells with electrophysiological properties and host synaptic connections following the intracerebral implantation of iPSCs derived from human fibroblasts ${ }^{100,101}$ are promising, but other studies using ST delivery of iPSCs have reported no functional improvement. ${ }^{102}$

\section{Stem cells and the immune system}

Transplanted stem cell survival may be influenced by host immune responses, but the transplanted cells may themselves modulate the host inflammatory microenvironment after stroke. The immunogenicity of allogeneic stem cells varies according to the expression of their major histocompatibility complex (MHC) I and II and other molecules that stimulate host CD8+ or CD4+ T cells. ${ }^{103}$ MSCs express very few MHC antigens, but cell surface marker expression may be modified by the host environment, and the lack of in vitro immunogenicity may not therefore be informative about the potential for problems in clinical use. However, to date, there have been no reports of cell-related adverse events or tumorigenesis following autologous MSC administration in the small number of early Phase I clinical trials in stroke ${ }^{104}$ and multiple sclerosis. ${ }^{105}$ Two clinical trials, of allogeneic $\mathrm{NSC}^{106}$ (NCT01151124) and $\mathrm{MSC}^{107}$ (NCT01297413) lines for the 
treatment of stroke with no coadministered immunosuppression, are currently investigating safety outcomes, including clinical, laboratory, and imaging markers. Although there is evidence that adult stem cells have an inherent immunologically privileged status and are capable of escaping rejection, ${ }^{108}$ it is unclear whether their MHC expression is altered by exposure to proinflammatory cytokines, such as occurs in ischemic tissue injury. ST-implanted neural progenitor cells have been observed to have low immunogenicity as they are not exposed to systemic immune surveillance, but the blood-brain barrier is damaged after stroke and the CNS probably does not retain this status. There are suggestions that low immunogenicity could be a unique property of NSCs, based on a lack in upregulation of the immunological response to transplantation of murine NSCs, and the lack of difference observed in animals, whether or not immunosuppressed, 2 weeks postimplantation and 4 weeks post-MCAo. ${ }^{109}$ Some xenogeneic animal stroke studies have coadministered immunosuppressant drugs on the assumption that the recipient species would reject donor cells of human origin. Whether or not xenogeneic studies necessitate immunosuppression is still unclear. Many studies have not reported the use of immunosuppression or have not considered studying its effects in detail..$^{36,40,45}$ Immunosuppressant drugs have independent neuroprotective effects in animal models of stroke, and their use was identified as a significant factor in modifying effect size estimates in a meta-analysis of animal studies. ${ }^{110}$

Transplanted stem cells initiate a dynamic sequence of host immunomodulatory actions on exposure to the host inflammatory microenvironment. They not only integrate and differentiate but also home in, extravasate into the CNS, and modulate immune responses in situ. ${ }^{111}$ NSCs are reported to show more tropism towards inflammatory sites than do MSCs. ${ }^{112}$ Both NSCs and MSCs exhibit host immune modulation in vivo. MSCs release neurotrophic factors, such as BDNF, provide trophic support for vulnerable neurons in the ischemic penumbra, support endogenous oligodendrogenesis, and regulate anti-inflammatory responses, leading to enhanced tissue sparing. ${ }^{65}$ NSCs attenuate brain inflammation, modulate microglia activation, limit demyelination, and promote host-driven repair. ${ }^{113}$

\section{Clinical trial design}

Ideally, preclinical evidence of efficacy, information on the optimal timing and mode of delivery, and toxicity (including tumorigenesis and possibly gene silencing studies) should be considered in clinical trial planning. The STEPS ${ }^{12,13}$ meetings have suggested essential minimum criteria for the design of cell therapy stroke trials, by incorporating general principles from the earlier STAIR proposals that primarily concerned pharmaceutical development. ${ }^{11}$ Although these recommend that preclinical studies include more than one strain of rodent, animals of varying ages, and that there be independent confirmation from one or more laboratories, in reality these recommendations are rarely followed due to high costs and potential commercial restrictions, and preclinical information may thus be limited.

The selection of an appropriate target stroke population will be influenced by the phase of study, expected mode of action of the cell therapy under study, and preclinical data. For studies primarily collecting safety data, chronic stroke patients with a broad range of severity who are not within the natural recovery period are likely to be candidates. The dose of stem cells for humans would usually be estimated based on animal studies and will need further human testing to define the maximum tolerated dose, minimum effective dose, and ideally, a doseresponse curve. For a safety trial, an ascending dose design could be incorporated, especially for ST-delivered cells. For studies gathering efficacy data, subjects are likely to be in the acute or subacute stage after stroke, having deficits that are measurable by well-validated clinical scales, and whose natural evolution and variability over time after stroke are understood. Biomarkers, such as imaging, may offer greater biological confidence in the effects of treatment, with sample sizes that are smaller than are necessary to distinguish differences in the clinical disability scales; imaging markers should correlate with clinically relevant measures. For long-term safety follow up, the prevalence of significant comorbidities in stroke populations and the intensity of observation in a typically disabled and elderly population need to be considered in order to minimize trial subject attrition.

Stroke lesion sizes and locations are heterogeneous, and there is considerable interindividual variation in the neuroanatomical systems involved. Experimental stroke induction is a more controlled event, intended to produce a consistent lesion size and distribution. Anatomical characterization will thus play a significant role in patient selection in trials, not only from the perspective of surgical planning and feasibility for studies using delivery by ST implantation, but also, more generally as a prognostic marker. For example, corticospinal tract integrity predicts motor impairment ${ }^{114}$ and the probability of motor recovery. ${ }^{115}$ Likewise, the timing of the ST intervention can be challenging in the acute stage, when lesion size varies considerably with improving edema and anatomical remodeling. While IV delivery is more straightforward from this aspect, a persistent occlusion of the target artery compromises IA cell delivery and may 
significantly reduce cell penetration or compromise survival at the target site. Timing will also be influenced by knowledge of the natural course of recovery and how this aligns with the chosen cell's mechanism of action to maximize effect.

The feasibility of blinding patients and trialists to treatment allocation varies by the treatment delivery route and the requirement for placebo controls. Both placebo and blinding are relatively easier for IV therapy than for more invasive delivery routes since there are procedural complications from either IA or direct intracerebral delivery. Whether the scientifically rigorous inclusion of placebo controls to permit double blinding is sufficiently justified instead of potentially less hazardous sham alternatives that could yield a single-blind study (for example, a small incision in the groin rather than an IA placebo injection, or a scalp incision or burr hole rather than an injection of placebo fluid into the brain) may depend on the stage of research and the procedural risks. Functional change, for example, serial clinical scores or functional brain imaging, including both pre- and post-treatment periods, may reduce variance. Domain-specific endpoints, as suggested by Cramer et al, ${ }^{116}$ may be more relevant than broad global outcome scales that traverse multiple neural systems; at the same time, they may restrict the trial entry criteria to those patients with very specific deficits, and thus reduce the generalizability of trial results (for example, motor outcome endpoints necessitate motor deficits at entry but a positive effect may arguably not be applicable to speech deficits). ${ }^{117}$ Imaging-based outcomes may help to compare metrics, as they could be applied to both humans and animals. The use of imaging biomarkers for recovery prediction is promising, and these are currently being tested. The major confound of providing routine physical rehabilitation treatment in stroke recovery trials is an unresolved issue. While some studies of therapy inputs for specific clinical problems have identified dose-response relationships, ${ }^{118,119}$ for many routinely applied interventions, the efficacy or dose relationship is unknown. Even where evidence supports the therapy interventions, few clinical services deliver the optimal dose routinely, and there is enormous variability across sites and healthcare systems. Since animal studies have reported that concomitant specific physical rehabilitation may be a prerequisite of stem cell efficacy, this represents a major challenge in clinical study design.

\section{Advances and future prospects in stem cell therapy for stroke}

Many clinical investigations are documented on trials databases as planned or underway (Table 1), but the great majority of these studies are safety and tolerability studies, with small sample sizes and unspecified control groups.
A wide range of cell types is being investigated, but most studies plan autologous bone marrow-derived cell administration by intravascular routes at subacute time points. These studies, if completed, will contribute valuable safety data that is a necessary prelude to large-scale efficacy trials, but ultimately, large randomized controlled trials with broad clinical endpoints will be required to judge the balance of risks and benefits.

The genetic modification of stem cells (for example to enhance the delivery of trophic factors, like $\mathrm{BDNF}^{120}$ or VEGF, ${ }^{121}$ or to address large scale manufacturing through conditional cell immortalization ${ }^{30}$ ) may offer advantages for allogeneic cell therapies. The allogeneic approaches offer the hypothetical advantage of immediate "off the shelf" availability, which is not possible with autologous cells, even if cells are not culture-expanded prior to administration. Laboratory research into the use of nonviral vectors for stable modification of cells, in vivo cell tracking, and the modification of stem cell gene expression profiles, is ongoing and will improve our understanding of cell function. ${ }^{122}$ Tissue replacement as a therapeutic goal is almost certainly beyond the scope of the current therapeutic approaches in stroke, but the development of extracellular matrix bioscaffolds, to provide structural support for human NSCs, is a promising and potentially relevant approach for chronic stroke and other forms of brain injury. ${ }^{123}$ The concept of stem cell-secreted extracellular membrane vesicles, providing extracellular waves of information capable of inducing multiple functional responses in adjacent and distant target cells, has emerged recently; the relevance of the bidirectional genetic information exchange between stem and target cells via MSC-secreted extracellular membrane vesicles ${ }^{124}$ is under investigation as a possible means of modifying graft-host interactions.

Parallel advances in biomaterial engineering and nanotechnology could provide an inert scaffold for ex-vivo stem cell expansion and intracranial delivery, ${ }^{125}$ and may in future address the limitation, for current cell therapy paradigms, of the major loss of brain tissue after stroke that leaves only a cystic cavity.

Applying novel imaging techniques to monitor stem cell effects and identify biomarkers is likely to be the key to the neurological application of cell therapies. Conventional structural imaging is unlikely to be helpful, but modalities, such as motor task fMRI can predict treatment response ${ }^{126}$ and provide a measure of the balance of interhemispheric control, ${ }^{127}$ and DTI can provide information on axonal integrity, which correlates with functional recovery. ${ }^{128}$ Approaches such as resting state fMRI may allow the 
assessment of the effect of stem cells at a network level on either hemisphere. ${ }^{129}$ Multimodal approaches ${ }^{130}$ combining fMRI and DTI are advancing, and more work with stem cell-treated subjects will improve the use of imaging-based biomarkers for patient selection, baseline stratification, and outcome assessment.

\section{Conclusion}

Contrary to long-held beliefs, we now know that the brain is highly malleable after an ischemic insult. Endogenous neurogenesis, angiogenesis, and synaptogenesis occurs in humans, albeit at a rate that is able to provide only partial functional recovery in the majority of cases. Cell therapy offers a potential for multimodal action that is promising within the domain of brain repair therapies. Despite the almost certain publication bias in animal stroke studies, stem cell experiments have shown evidence of cell migration to the lesion, survival, and varying degrees of differentiation. Both tissue-specific NSCs and non-tissue-specific MSCs have been associated with significantly improved behavioral outcomes. A comprehensive understanding of their mechanism of action is lacking, but tissue replacement is now believed likely to constitute only a minor contribution (if any) to the therapeutic effect. Accordingly, a cell type's capacity to differentiate along specific pathways is likely to be a less relevant consideration. The multiple mechanisms of action of stem cells include the secretion of trophic factors, immunomodulation, and anti-inflammatory effects. The great majority of the early cell therapy clinical studies have involved adult-derived cells of either autologous or allogeneic origin, and no major safety issues have been identified to date, although the numbers of subjects have been extremely small and follow-up periods limited. Several clinical trials are ongoing or planned, mostly using MSC cells delivered by IV infusion.

\section{Disclosure}

KWM is the chief investigator of the PISCES trial of human neural stem cells in stroke and a related Phase II trial. Both trials are funded by ReNeuron Ltd. DK is a subinvestigator for the PISCES trial. The authors report no other conflicts of interest.

\section{References}

1. Norrving B, Kissela B. The global burden of stroke and need for a continuum of care. Neurology. 2013;80(3 Suppl 2):S5-S12.

2. Truelsen T, Heuschmann PU, Bonita R, et al. Standard method for developing stroke registers in low-income and middle-income countries: experiences from a feasibility study of a stepwise approach to stroke surveillance (STEPS Stroke). Lancet Neurol. 2007;6(2):134-139.
3. Donnan GA, Fisher M, Macleod M, Davis SM. Stroke. Lancet. 2008;371(9624):1612-1623.

4. Rha JH, Saver JL. The impact of recanalization on ischemic stroke outcome: a meta-analysis. Stroke. 2007;38(3):967-973.

5. Hacke W, Donnan G, Fieschi C, et al; ATLANTIS Trials Investigators; ECASS Trials Investigators; NINDS rt-PA Study Group Investigators. Association of outcome with early stroke treatment: pooled analysis of ATLANTIS, ECASS, and NINDS rt-PA stroke trials. Lancet. 2004;363(9411):768-774.

6. Langhorne P, Bernhardt J, Kwakkel G. Stroke rehabilitation. Lancet. 2011;377(9778):1693-1702.

7. Ginsberg MD. Neuroprotection for ischemic stroke: past, present and future. Neuropharmacology. 2008;55(3):363-389.

8. Muir KW. Heterogeneity of stroke pathophysiology and neuroprotective clinical trial design. Stroke. 2002;33(6):1545-1550.

9. Macleod MR, O'Collins T, Howells DW, Donnan GA. Pooling of animal experimental data reveals influence of study design and publication bias. Stroke. 2004;35(5):1203-1208.

10. Dirnagl U, Iadecola C, Moskowitz MA. Pathobiology of ischaemic stroke: an integrated view. Trends Neurosci. 1999;22(9):391-397.

11. Stroke Therapy Academic Industry Roundtable (STAIR). Recommendations for standards regarding preclinical neuroprotective and restorative drug development. Stroke. 1999;30(12):2752-2758.

12. Stem Cell Therapies as an Emerging Paradigm in Stroke Participants. Stem Cell Therapies as an Emerging Paradigm in Stroke (STEPS): bridging basic and clinical science for cellular and neurogenic factor therapy in treating stroke. Stroke. 2009;40(2):510-515.

13. Savitz SI, Chopp M, Deans R, Carmichael ST, Phinney D, Wechsler L. Stem Cell Therapy as an Emerging Paradigm for Stroke (STEPS) II. Stroke. 2011;42(3):825-829.

14. Altman J, Das GD. Post-natal origin of microneurones in the rat brain. Nature. 1965;207(5000):953-956.

15. Gage FH. Mammalian neural stem cells. Science. 2000;287(5457): 1433-1438.

16. Wang C, Liu F, Liu YY, et al. Identification and characterization of neuroblasts in the subventricular zone and rostral migratory stream of the adult human brain. Cell Res. 2011;21(11):1534-1550.

17. Eriksson PS, Perfilieva E, Björk-Eriksson T, et al. Neurogenesis in the adult human hippocampus. Nat Med. 1998;4(11):1313-1317.

18. Seri B, Herrera DG, Gritti A, et al. Composition and organization of the SCZ: a large germinal layer containing neural stem cells in the adult mammalian brain. Cereb Cortex. 2006;16 Suppl 1:i103-i111.

19. Shimada IS, Peterson BM, Spees JL. Isolation of locally derived stem/progenitor cells from the peri-infarct area that do not migrate from the lateral ventricle after cortical stroke. Stroke. 2010;41(9): e552-e560.

20. Lindvall O, Kokaia Z, Martinez-Serrano A. Stem cell therapy for human neurodegenerative disorders-how to make it work. Nat Med. 2004;10 Suppl:S42-S50.

21. Jin K, Wang X, Xie L, et al. Evidence for stroke-induced neurogenesis in the human brain. Proc Natl Acad Sci U S A. 2006;103(35): 13198-13202.

22. Shang J, Deguchi K, Ohta Y, et al. Strong neurogenesis, angiogenesis, synaptogenesis, and antifibrosis of hepatocyte growth factor in rats brain after transient middle cerebral artery occlusion. J Neurosci Res. 2011;89(1):86-95.

23. Chen J, Zhang ZG, LiY, et al. Statins induce angiogenesis, neurogenesis, and synaptogenesis after stroke. Ann Neurol. 2003;53(6):743-751.

24. Zhao C, Deng W, Gage FH. Mechanisms and functional implications of adult neurogenesis. Cell. 2008;132(4):645-660.

25. Arvidsson A, Collin T, Kirik D, Kokaia Z, Lindvall O. Neuronal replacement from endogenous precursors in the adult brain after stroke. Nat Med. 2002;8(9):963-970.

26. Cramer SC, Chopp M. Recovery recapitulates ontogeny. Trends Neurosci. 2000;23(6):265-271.

27. Shyu WC, Liu DD, Lin SZ, et al. Implantation of olfactory ensheathing cells promotes neuroplasticity in murine models of stroke. J Clin Invest. 2008;118(7):2482-2495. 
28. Choi D, Gladwin K. Olfactory ensheathing cells, part II: source of cells and application to patients. World Neurosurg. Epub July 24, 2013.

29. Peschanski M, Defer G, N'Guyen JP, et al. Bilateral motor improvement and alteration of L-dopa effect in two patients with Parkinson's disease following intrastriatal transplantation of foetal ventral mesencephalon. Brain 1994;117(3):487-499.

30. Takahashi K, Yasuhara T, Shingo T, et al. Embryonic neural stem cells transplanted in middle cerebral artery occlusion model of rats demonstrated potent therapeutic effects, compared to adult neural stem cells. Brain Res. 2008;1234:172-182.

31. Pollock K, Stroemer P, Patel S, et al. A conditionally immortal clonal stem cell line from human cortical neuroepithelium for the treatment of ischemic stroke. Exp Neurol. 2006;199(1):143-155.

32. Massirer KB, Carromeu C, Griesi-Oliveira K, Muotri AR. Maintenance and differentiation of neural stem cells. Wiley Interdiscip Rev Syst Biol Med. 2011;3(1):107-114.

33. De Filippis L, Delia D. Hypoxia in the regulation of neural stem cells. Cell Mol Life Sci. 2011;68(17):2831-2844.

34. Cacci E, Villa A, Parmar M, et al. Generation of human cortical neurons from a new immortal fetal neural stem cell line. Exp Cell Res. 2007;313(3):588-601.

35. Stevanato L, Corteling RL, Stroemer P, et al. c-MycERTAM transgene silencing in a genetically modified human neural stem cell line implanted into MCAo rodent brain. BMC Neurosci. 2009;10:86.

36. Andres RH, Horie N, Slikker W, et al. Human neural stem cells enhance structural plasticity and axonal transport in the ischaemic brain. Brain. 2011;134(Pt 6): 1777-1789.

37. Ishibashi S, Sakaguchi M, Kuroiwa T, et al. Human neural stem/ progenitor cells, expanded in long-term neurosphere culture, promote functional recovery after focal ischemia in Mongolian gerbils. J Neurosci Res. 2004;78(2):215-223.

38. Darsalia V, Allison SJ, Cusulin C, et al. Cell number and timing of transplantation determine survival of human neural stem cell grafts in stroke-damaged rat brain. J Cereb Blood Flow Metab. 2011;31(1): $235-242$.

39. Rosenblum S, Wang N, Smith TN, et al. Timing of intra-arterial neural stem cell transplantation after hypoxia-ischemia influences cell engraftment, survival, and differentiation. Stroke. 2012;43(6):1624-1631.

40. Chu K, Park KI, Lee ST, et al. Combined treatment of vascular endothelial growth factor and human neural stem cells in experimental focal cerebral ischemia. Neurosci Res. 2005;53(4):384-390.

41. Jiang Q, Zhang ZG, Ding GL, et al. MRI detects white matter reorganization after neural progenitor cell treatment of stroke. Neuroimage. 2006;32(3):1080-1089.

42. Kelly S, Bliss TM, Shah AK, et al. Transplanted human fetal neural stem cells survive, migrate, and differentiate in ischemic rat cerebral cortex. Proc Natl Acad Sci U SA. 2004;101(32):11839-11844.

43. Pendharkar AV, Chua JY, Andres RH, et al. Biodistribution of neural stem cells after intravascular therapy for hypoxic-ischemia. Stroke. 2010;41(9):2064-2070.

44. Li L, Jiang Q, Ding G, et al. Effects of administration route on migration and distribution of neural progenitor cells transplanted into rats with focal cerebral ischemia, an MRI study. J Cereb Blood Flow Metab. 2010;30(3):653-662.

45. Daadi MM, Li Z, Arac A, et al. Molecular and magnetic resonance imaging of human embryonic stem cell-derived neural stem cell grafts in ischemic rat brain. Mol Ther. 2009;17(7):1282-1291.

46. Zhang P, Li J, Liu Y, et al. Human neural stem cell transplantation attenuates apoptosis and improves neurological functions after cerebral ischemia in rats. Acta Anaesthesiol Scand. 2009;53(9):1184-1191.

47. Song M, Kim YJ, Kim YH, Roh J, Kim SU, Yoon BW. Effects of duplicate administration of human neural stem cell after focal cerebral ischemia in the rat. Int J Neurosci. 2011;121(8):457-461.

48. Hicks A, Schallert T, Jolkkonen J. Cell-based therapies and functional outcome in experimental stroke. Cell Stem Cell. 2009;5(2): 139-140.

49. Beaulieu C. The basis of anisotropic water diffusion in the nervous system - a technical review. NMR Biomed. 2002;15(7-8):435-455.
50. Friedenstein AJ, Petrakova KV, Kurolesova AI, Frolova GP. Heterotopic of bone marrow. Analysis of precursor cells for osteogenic and hematopoietic tissues. Transplantation. 1968;6(2):230-247.

51. Horwitz EM, Le Blanc K, Dominici M, et al; International Society for Cellular Therapy. Clarification of the nomenclature for MSC: The International Society for Cellular Therapy position statement. Cytotherapy. 2005;7(5):393-395.

52. Dominici M, Le Blanc K, Mueller I, et al. Minimal criteria for defining multipotent mesenchymal stromal cells. The International Society for Cellular Therapy position statement. Cytotherapy. 2006;8(4):315-317.

53. Xu H, Miki K, Ishibashi S, et al. Transplantation of neuronal cells induced from human mesenchymal stem cells improves neurological functions after stroke without cell fusion. J Neurosci Res. 2010;88(16): 3598-3609.

54. Gutiérrez-Fernández M, Rodríguez-Frutos B, Alvarez-Grech J, et al. Functional recovery after hematic administration of allogenic mesenchymal stem cells in acute ischemic stroke in rats. Neuroscience. 2011;175:394-405.

55. Cui X, Chen J, Zacharek A, et al. Nitric oxide donor upregulation of stromal cell-derived factor-1/chemokine (CXC motif) receptor 4 enhances bone marrow stromal cell migration into ischemic brain after stroke. Stem Cells. 2007;25(11):2777-2785.

56. Chen ZZ, Jiang XD, Zhang LL, et al. Beneficial effect of autologous transplantation of bone marrow stromal cells and endothelial progenitor cells on cerebral ischemia in rabbits. Neurosci Lett. 2008;445(1):36-41.

57. Horita Y, Honmou O, Harada K, Houkin K, Hamada H, Kocsis JD. Intravenous administration of glial cell line-derived neurotrophic factor gene-modified human mesenchymal stem cells protects against injury in a cerebral ischemia model in the adult rat. $J$ Neurosci Res. 2006;84(7): $1495-1504$.

58. Ruan GP, Han YB, Wang TH, et al. Comparative study among three different methods of bone marrow mesenchymal stem cell transplantation following cerebral infarction in rats. Neurol Res. 2013;35(2): 212-220.

59. Braun RG, Andrews EM, Kartje GL. Kinematic analysis of motor recovery with human adult bone marrow-derived somatic cell therapy in a rat model of stroke. Neurorehabil Neural Repair. 2012;26(7):898-906.

60. Shen LH, LiY, Chen J, et al. Therapeutic benefit of bone marrow stromal cells administered 1 month after stroke. J Cereb Blood Flow Metab. 2007;27(1):6-13.

61. Rosenkranz K, Kumbruch S, Lebermann K, et al. The chemokine SDF-1/CXCL12 contributes to the 'homing' of umbilical cord blood cells to a hypoxic-ischemic lesion in the rat brain. $J$ Neurosci Res. 2010;88(6):1223-1233.

62. Kang SK, Lee DH, Bae YC, Kim HK, Baik SY, Jung JS. Improvement of neurological deficits by intracerebral transplantation of human adipose tissue-derived stromal cells after cerebral ischemia in rats. Exp Neurol. 2003;183(2):355-366.

63. Kim SS, Yoo SW, Park TS, et al. Neural induction with neurogenin 1 increases the therapeutic effects of mesenchymal stem cells in the ischemic brain. Stem Cells. 2008;26(9):2217-2228.

64. Mora-Lee S, Sirerol-Piquer MS, Gutiérrez-Pérez M, et al. Therapeutic effects of hMAPC and hMSC transplantation after stroke in mice. PLoS One. 2012;7(8):e43683.

65. Chen J, Li Y, Wang L, et al. Therapeutic benefit of intravenous administration of bone marrow stromal cells after cerebral ischemia in rats. Stroke. 2001;32(4):1005-1011

66. Chopp M, Li Y. Treatment of neural injury with marrow stromal cells. Lancet Neurol. 2002;1(2):92-100.

67. Wakabayashi K, Nagai A, Sheikh AM, et al. Transplantation of human mesenchymal stem cells promotes functional improvement and increased expression of neurotrophic factors in a rat focal cerebral ischemia model. J Neurosci Res. 2010;88(5):1017-1025.

68. Wu Y, Chen L, Scott PG, Tredget EE. Mesenchymal stem cells enhance wound healing through differentiation and angiogenesis. Stem Cells. 2007;25(10):2648-2659. 
69. Li J, Zhu H, Liu Y, et al. Human mesenchymal stem cell transplantation protects against cerebral ischemic injury and upregulates interleukin-10 expression in Macacafascicularis. Brain Res. 2010;1334:65-72.

70. Sidoryk-Wegrzynowicz M, Wegrzynowicz M, Lee E, Bowman AB, Aschner M. Role of astrocytes in brain function and disease. Toxicol Pathol. 2011;39(1):115-123.

71. Sofroniew MV. Reactive astrocytes in neural repair and protection. Neuroscientist. 2005;11(5):400-407.

72. Gao Q, Li Y, Chopp M. Bone marrow stromal cells increase astrocyte survival via upregulation of phosphoinositide 3-kinase/ threonine protein kinase and mitogen-activated protein kinase kinase/ extracellular signal-regulated kinase pathways and stimulate astrocyte trophic factor gene expression after anaerobic insult. Neuroscience. 2005;136(1):123-134.

73. Shen LH, LiY, Chen J, et al. Intracarotid transplantation of bone marrow stromal cells increases axon-myelin remodeling after stroke. Neuroscience. 2006;137(2):393-399.

74. Andrews EM, Tsai SY, Johnson SC, et al. Human adult bone marrowderived somatic cell therapy results in functional recovery and axonal plasticity following stroke in the rat. Exp Neurol. 2008;211(2): 588-592.

75. Bao X, Wei J, Feng M, et al. Transplantation of human bone marrowderived mesenchymal stem cells promotes behavioral recovery and endogenous neurogenesis after cerebral ischemia in rats. Brain Res. 2011;1367:103-113.

76. Bao X, Feng M, Wei J, et al. Transplantation of Flk-1+ human bone marrow-derived mesenchymal stem cells promotes angiogenesis and neurogenesis after cerebral ischemia in rats. Eur J Neurosci. 2011;34(1):87-98.

77. Honma $\mathrm{T}$, Honmou $\mathrm{O}$, Iihoshi $\mathrm{S}$, et al. Intravenous infusion of immortalized human mesenchymal stem cells protects against injury in a cerebral ischemia model in adult rat. Exp Neurol. 2006;199(1):56-66.

78. Kurozumi K, Nakamura K, Tamiya T, et al. Mesenchymal stem cells that produce neurotrophic factors reduce ischemic damage in the rat middle cerebral artery occlusion model. Mol Ther. 2005;11(1):96-104.

79. Koh SH, Kim KS, Choi MR, et al. Implantation of human umbilical cord-derived mesenchymal stem cells as a neuroprotective therapy for ischemic stroke in rats. Brain Res. 2008;1229:233-248.

80. Chen J, Sanberg PR, Li Y, et al. Intravenous administration of human umbilical cord blood reduces behavioral deficits after stroke in rats. Stroke. 2001;32(11):2682-2688.

81. Borlongan CV, Hadman M, Sanberg CD, Sanberg PR. Central nervous system entry of peripherally injected umbilical cord blood cells is not required for neuroprotection in stroke. Stroke. 2004;35(10): 2385-2389.

82. Willing AE, Lixian J, Milliken M, et al. Intravenous versus intrastriatal cord blood administration in a rodent model of stroke. J Neurosci Res. 2003;73(3):296-307.

83. Taguchi A, Soma T, Tanaka H, et al. Administration of CD34+ cells after stroke enhances neurogenesis via angiogenesis in a mouse model. J Clin Invest. 2004;114(3):330-338.

84. Yoder MC. Human endothelial progenitor cells. Cold Spring Harb Perspect Med. 2012;2(7):1-14.

85. Iskander A, Knight RA, Zhang ZG, et al. Intravenous administration of human umbilical cord blood-derived AC133+ endothelial progenitor cells in rat stroke model reduces infarct volume: magnetic resonance imaging and histological findings. Stem Cells Transl Med. 2013;2(9):703-714.

86. Thomson JA, Itskovitz-Eldor J, Shapiro SS, et al. Embryonic stem cell lines derived from human blastocysts. Science. 1998;282(5391): $1145-1147$

87. Ben-David U, Benvenisty N. The tumorigenicity of human embryonic and induced pluripotent stem cells. Nat Rev Cancer. 2011;11: 268-277.

88. Seminatore C, Polentes J, Ellman D, et al. The postischemic environment differentially impacts teratoma or tumor formation after transplantation of human embryonic stem cell-derived neural progenitors. Stroke. 2010;41(1):153-159.
89. Azevedo-Pereira RL, Daadi MM. Isolation and purification of selfrenewable human neural stem cells for cell therapy in experimental model of ischemic stroke. Methods Mol Biol. 2013;1059:157-167.

90. Drury-Stewart D, Song M, Mohamad O, et al. Highly efficient differentiation of neural precursors from human embryonic stem cells and benefits of transplantation after ischemic stroke in mice. Stem Cell Res Ther. 2013;4(4):93.

91. Bühnemann C, Scholz A, Bernreuther C, et al. Neuronal differentiation of transplanted embryonic stem cell-derived precursors in stroke lesions of adult rats. Brain. 2006;129(Pt 12):3238-3248.

92. Daadi MM, Maag AL, Steinberg GK. Adherent self-renewable human embryonic stem cell-derived neural stem cell line: functional engraftment in experimental stroke model. PLoS One. 2008;3(2): e1644.

93. Reyes JH, O'Shea KS, Wys NL, et al. Glutamatergic neuronal differentiation of mouse embryonic stem cells after transient expression of neurogenin 1 and treatment with BDNF and GDNF: in vitro and in vivo studies. $J$ Neurosci. 2008;28(48):12622-12631.

94. Antonic A, Sena ES, Donnan GA, Howells DW. Human in vitro models of ischemic stroke: a test bed for translation. Transl Stroke Res. 2012;3:306-309.

95. Yu J, Vodyanik MA, Smuga-Otto K, et al. Induced pluripotent stem cell lines derived from human somatic cells. Science. 2007; 318(5858):1917-1920.

96. Takahashi K, Yamanaka S. Induction of pluripotent stem cells from mouse embryonic and adult fibroblast cultures by defined factors. Cell. 2006;126(4):663-676.

97. Yamanaka S, Blau HM. Nuclear reprogramming to a pluripotent state by three approaches. Nature. 2010;465(7299):704-712.

98. Malik N, Rao MS. A review of the methods for human iPSC derivation. Methods Mol Biol. 2013;997:23-33.

99. Kawai H, Yamashita T, Ohta Y, et al. Tridermal tumorigenesis of induced pluripotent stem cells transplanted in ischemic brain. J Cereb Blood Flow Metab. 2010;30(8):1487-1493.

100. Oki K, Tatarishvili J, Wood J, et al. Human-induced pluripotent stem cells form functional neurons and improve recovery after grafting in stroke-damaged brain. Stem Cells. 2012;30(6):1120-1133.

101. Jiang M, Lv L, Ji H, et al. Induction of pluripotent stem cells transplantation therapy for ischemic stroke. Mol Cell Biochem. 2011;354(1-2): $67-75$.

102. Jensen MB, Yan H, Krishnaney-Davison R, Al Sawaf A, Zhang SC. Survival and differentiation of transplanted neural stem cells derived from human induced pluripotent stem cells in a rat stroke model. J Stroke Cerebrovasc Dis. 2013;22(4):304-308.

103. Pluchino S, Cossetti C. How stem cells speak with host immune cells in inflammatory brain diseases. Glia. 2013;61(9):1379-1401.

104. Bhasin A, Srivastava MV, Kumaran SS, et al. Autologous mesenchymal stem cells in chronic stroke. Cerebrovasc Dis Extra. 2011;1(1):93-104.

105. Connick P, Kolappan M, Crawley C, et al. Autologous mesenchymal stem cells for the treatment of secondary progressive multiple sclerosis: an open-label phase 2a proof-of-concept study. Lancet Neurol. 2012;11(2):150-156.

106. ReNeuron Limited. Pilot Investigation of Stem Cells in Stroke (PISCES). In: ClinicalTrials.gov [website on the Internet]. Bethesda, MD: US National Library of Medicine; 2010 [updated March 27, 2013]. Available from: http://clinicaltrials.gov/ct2/show/NCT01151124. NLM identifier: NCT01151124. Accessed November 20, 2013.

107. Stemedica Cell Technologies, Inc. A Study of Allogeneic Mesenchymal Bone Marrow Cells in Subjects With Ischemic Stroke. In: ClinicalTrials.gov [website on the Internet]. Bethesda, MD: US National Library of Medicine; 2011 [updated December 13, 2012]. Available from: http://clinicaltrials.gov/ct2/show/NCT01297413?term $=$ NCT01297413\&rank=1. NLM identifier: NCT01297413. Accessed November 20, 2013.

108. Bifari F, Pacelli L, Krampera M. Immunological properties of embryonic and adult stem cells. World J Stem Cells. 2010;2(3): $50-60$ 
109. Modo M, Rezaie P, Heuschling P, Patel S, Male DK, Hodges H. Transplantation of neural stem cells in a rat model of stroke: assessment of short-term graft survival and acute host immunological response. Brain Res. 2002;958(1):70-82.

110. Lees JS, Sena ES, Egan KJ, et al. Stem cell-based therapy for experimental stroke: a systematic review and meta-analysis. Int $J$ Stroke. 2012;7(7):582-588

111. Kokaia Z, Martino G, Schwartz M, Lindvall O. Cross-talk between neural stem cells and immune cells: the key to better brain repair? Nat Neurosci. 2012;15(8):1078-1087.

112. Martino G, Pluchino S. The therapeutic potential of neural stem cells. Nat Rev Neurosci. 2006;7(5):395-406.

113. Martino G, Pluchino S, Bonfanti L, Schwartz M. Brain regeneration in physiology and pathology: the immune signature driving therapeutic plasticity of neural stem cells. Physiol Rev. 2011;91(4): $1281-1304$

114. Lindenberg R, Renga V, Zhu LL, Betzler F, Alsop D, Schlaug G. Structural integrity of corticospinal motor fibers predicts motor impairment in chronic stroke. Neurology. 2010;74(4): 280-287.

115. Sterr A, Shen S, Szameitat AJ, Herron KA. The role of corticospinal tract damage in chronic motor recovery and neurorehabilitation: a pilot study. Neurorehabil Neural Repair. 2010;24(5): 413-419.

116. Cramer SC, Koroshetz WJ, Finklestein SP. The case for modalityspecific outcome measures in clinical trials of stroke recoverypromoting agents. Stroke. 2007;38(4):1393-1395.

117. Quinn TJ, Dawson J, Walters MR, Lees KR. Functional outcome measures in contemporary stroke trials. Int J Stroke. 2009;4(3): 200-205.

118. Cooke EV, Mares K, Clark A, Tallis RC, Pomeroy VM. The effects of increased dose of exercise-based therapies to enhance motor recovery after stroke: a systematic review and meta-analysis. BMC Med. 2010;8:60.

119. French B, Thomas L, Leathley M, et al. Does repetitive task training improve functional activity after stroke? A Cochrane systematic review and meta-analysis. J Rehabil Med. 2010;42(1):9-14.

120. Kurozumi K, Nakamura K, Tamiya T, et al. BDNF gene-modified mesenchymal stem cells promote functional recovery and reduce infarct size in the rat middle cerebral artery occlusion model. Mol Ther. 2004;9(2):189-197.

121. Toyama K, Honmou O, Harada K, et al. Therapeutic benefits of angiogenetic gene-modified human mesenchymal stem cells after cerebral ischemia. Exp Neurol. 2009;216(1):47-55.

122. Fontes A, Lakshmipathy U. Advances in genetic modification of pluripotent stem cells. Biotechnol Adv. 2013;31(7): 994-1001.

123. Bible E, Dell'Acqua F, Solanky B, et al. Non-invasive imaging of transplanted human neural stem cells and ECM scaffold remodeling in the stroke-damaged rat brain by (19)F- and diffusion-MRI. Biomaterials. 2012;33(10):2858-2871.

124. Huang YC, Parolini O, Deng L. The potential role of microvesicles in mesenchymal stem cell-based therapy. Stem Cells Dev. 2013;22(6):841-844.

125. Chai C, Leong KW. Biomaterials approach to expand and direct differentiation of stem cells. Mol Ther. 2007;15(3):467-480.

126. Cramer SC, Parrish TB, Levy RM, et al. Predicting functional gains in a stroke trial. Stroke. 2007;38(7):2108-2114.

127. Cramer SC, Nelles G, Benson RR, et al. A functional MRI study of subjects recovered from hemiparetic stroke. Stroke. 1997;28(12): 2518-2527.

128. Stinear CM, Barber PA, Smale PR, Coxon JP, Fleming MK, Byblow WD. Functional potential in chronic stroke patients depends on corticospinal tract integrity. Brain. 2007;130(Pt 1): 170-180.

129. Carter AR, Shulman GL, Corbetta M. Why use a connectivity-based approach to study stroke and recovery of function? Neuroimage. 2012;62(4):2271-2280
130. Wang LE, Tittgemeyer M, Imperati D, et al. Degeneration of corpus callosum and recovery of motor function after stroke: a multimodal magnetic resonance imaging study. Hum Brain Mapp. 2012;33(12): 2941-2956

131. Federal University of Rio de Janeiro. Study of Autologous Stem Cell Transplantation for Patients With Ischemic Stroke. In: ClinicalTrials.gov [website on the Internet]. Bethesda, MD: US National Library of Medicine; 2007 [updated May 18, 2011]. Available from: http://clinicaltrials. gov/ct2/show/NCT00473057?term=NCT00473057\&rank=1. NLM identifier: NCT00473057. Accessed November 20, 2013.

132. Manipal Acunova Ltd. Intravenous Autologous Bone Marrow-derived Stem Cells Therapy for Patients With Acute Ischemic Stroke. In: ClinicalTrials.gov [website on the Internet]. Bethesda, MD: US National Library of Medicine; 2011. Available from: http://clinicaltrials.gov/ct2/ show/NCT01501773?term=NCT01501773\&rank=1. NLM identifier: NCT01501773. Accessed November 20, 2013.

133. Chaitanya Hospital, Pune. A Clinical Trial to Study the Safety and Efficacy of Bone Marrow Derived Autologous Cell for the Treatment of Stroke. (BMACS). In: ClinicalTrials.gov [website on the Internet] Bethesda, MD: US National Library of Medicine; 2013 [updated April 12, 2013]. Available from: http://clinicaltrials.gov/ct2/show/NCT0183 2428?term=NCT01832428\&rank=1. NLM identifier: NCT01832428. Accessed November 20, 2013.

134. National University of Malaysia. Intravenous Autologous Mesenchymal Stem Cells Transplantation to Treat Middle Cerebral Artery Infarct. In: ClinicalTrials.gov [website on the Internet]. Bethesda, MD: US National Library of Medicine; 2011 [updated October 26, 2011]. Available from: http://clinicaltrials.gov/ct2/show/NCT01461720?term=NCT0146172 0\&rank=1. NLM identifier: NCT01461720. Accessed November 20, 2013.

135. University of California, Irvine. Safety of Escalating Doses of Intravenous Bone Marrow-Derived Mesenchymal Stem Cells in Patients With a New Ischemic Stroke. In: ClinicalTrials.gov [website on the Internet]. Bethesda, MD: US National Library of Medicine; 2013 [updated May 8, 2013]. Available from: http://clinicaltrials.gov/show/ NCT01849887. NLM identifier: NCT01849887. Accessed November 20, 2013.

136. Wenzhou Medical University. Autologous Bone Marrow Mesenchymal Stem Cell Transplantation for Chronic Stroke. In: ClinicalTrials gov [website on the Internet]. Bethesda, MD: US National Library of Medicine; 2012 [updated October 22, 2012]. Available from: http:/ clinicaltrials.gov/show/NCT01714167. NLM identifier: NCT01714167. Accessed November 20, 2013.

137. Samsung Medical Center. The STem Cell Application Researches and Trials In NeuroloGy-2 (STARTING-2) Study. In: ClinicalTrials.gov [website on the Internet]. Bethesda, MD: US National Library of Medicine; 2012 [updated August 11, 2013]. Available from: http://clinicaltrials.gov/ct2/ show/NCT01716481?term=NCT01716481\&rank=1. NLM identifier: NCT01716481. Accessed November 20, 2013.

138. University Hospital, Grenoble. Intravenous Stem Cells After Ischemic Stroke (ISIS). In: ClinicalTrials.gov [website on the Internet]. Bethesda, MD: US National Library of Medicine; 2009 [updated September 4, 2013]. Available from: http://clinicaltrials.gov/ct2/show/NCT00875654?t erm=NCT00875654\&rank=1. NLM identifier: NCT00875654. Accessed November 20, 2013.

139. Hospital Universitario Central de Asturias. Autologous Bone Marrow Stem Cells in Middle Cerebral Artery Acute Stroke Treatment. In: ClinicalTrials.gov [website on the Internet]. Bethesda, MD: US National Library of Medicine; 2008 [updated November 27, 2011]. Available from: http://clinicaltrials.gov/ct2/show/NCT00761982?term=NCT007 61982\&rank=1. NLM identifier: NCT00761982. Accessed November 20, 2013.

140. Imperial College London. Autologous Bone Marrow Stem Cells in Ischemic Stroke. In: ClinicalTrials.gov [website on the Internet]. Bethesda, MD: US National Library of Medicine; 2007 [updated July 2, 2012]. Available from: http://clinicaltrials.gov/ct2/show/NCT00535197?term $=$ NCT00535197\&rank=1. NLM identifier: NCT00535197. Accessed November 20, 2013. 
141. Celgene Corporation. Study of Human Placenta-derived Cells (PDA001) to Evaluate the Safety and Effectiveness for Patients With Ischemic Stroke. In: ClinicalTrials.gov [website on the Internet]. Bethesda, MD: US National Library of Medicine; 2011 [updated August 12, 2013]. Available from: http://clinicaltrials.gov/ct2/show/NCT01310114?term =NCT01310114\&rank=1. NLM identifier: NCT01310114. Accessed November 20, 2013.

142. Ageless Regenerative Institute. Study to Assess the Safety and Effects of Autologous Adipose-Derived Stromal Cells in Patients After Stroke. In: ClinicalTrials.gov [website on the Internet]. Bethesda, MD: US National Library of Medicine; 2011 [updated October 28, 2013]. Available from: http://clinicaltrials.gov/ct2/show/NCT01453829?term=NCT0145382 9\&rank=1. NLM identifier: NCT01453829. Accessed November 20, 2013.

143. Instituto de Investigación Hospital Universitario La Paz. Reparative Therapy in Acute Ischemic Stroke With Allogenic Mesenchymal Stem Cells From Adipose Tissue, Safety Assessment, a Randomised, Double Blind Placebo Controlled Single Center Pilot Clinical Trial (AMASCIS-01). In: ClinicalTrials.gov [website on the Internet]. Bethesda, MD: US National Library of Medicine; 2012[updated September 6, 2013]. Available from: http://clinicaltrials.gov/ct2/show/NCT01678 534?term=NCT01678534\&rank=1.NLM identifier: NCT01678534. Accessed November 20, 2013.

144. The University of Texas Health Science Center, Houston. Safety/ Feasibility of Autologous Mononuclear Bone Marrow Cells in Stroke Patients. In: ClinicalTrials.gov [website on the Internet]. Bethesda, MD: US National Library of Medicine; 2009 [updated May 4, 2012]. Available from: http://clinicaltrials.gov/ct2/show/NCT00859014?term =NCT00859014\&rank=1. NLM identifier: NCT00859014. Accessed November 20, 2013.

145. Zhejiang Hospital. Autologous Hematopoietic Stem Cell Transplantation in Ischemic Stroke (AHSCTIS). In: ClinicalTrials.gov [website on the Internet]. Bethesda, MD: US National Library of Medicine; 2012 [updated January 24, 2012]. Available from: http://clinicaltrials. gov/ct2/show/NCT01518231?term=NCT01518231\&rank=1. NLM identifier: NCT01518231. Accessed November 20, 2013.
146. China Medical University Hospital. Study of Purified Umbilical Cord Blood CD34+ Stem Cell on Chronic Ischemic Stroke. In: ClinicalTrials.gov [website on the Internet]. Bethesda, MD: US National Library of Medicine; 2011 [updated July 31, 2012]. Available from: http://clinicaltrials.gov/ct2/show/NCT01438593?term= NCT01438593\&rank=1. NLM identifier: NCT01438593. Accessed November 20, 2013.

147. Athersys, Inc. Study to Examine the Effects of MultiStem in Ischemic Stroke. In: ClinicalTrials.gov [website on the Internet]. Bethesda, MD: US National Library of Medicine; 2011 [updated August 22, 2013]. Available from: http://clinicaltrials.gov/ct2/show/NCT01436487?term $=$ NCT01436487\&rank=1. NLM identifier: NCT01436487. Accessed November 20, 2013.

148. Aldagen. Study of ALD-401 Via Intracarotid Infusion in Ischemic Stroke Subjects. In: ClinicalTrials.gov [website on the Internet]. Bethesda, MD: US National Library of Medicine; 2011 [updated July 29, 2013]. Available from: http://clinicaltrials.gov/ct2/show/NCT0127 3337?term=NCT01273337\&rank=1. NLM identifier: NCT01273337. Accessed November 20, 2013.

149. SanBio, Inc. A Study of Modified Stem Cells in Stable Ischemic Stroke. In: ClinicalTrials.gov [website on the Internet]. Bethesda, MD: US National Library of Medicine; 2011 [updated August 30, 2013]. Available from: http://clinicaltrials.gov/ct2/show/NCT01287936?term $=$ NCT01287936\&rank=1. NLM identifier: NCT01287936. Accessed November 20, 2013.

150. Southern Medical University, China. Autologous Bone Marrow Stromal Cell and Endothelial Progenitor Cell Transplantation in Ischemic Stroke (AMETIS). In: ClinicalTrials.gov [website on the Internet]. Bethesda, MD: US National Library of Medicine; 2011 [updated November 8, 2011]. Available from: http://clinicaltrials. gov/ct2/show/NCT01468064?term=NCT01468064\&rank=1.NLM identifier: NCT01468064. Accessed November 20, 2013.
Stem Cells and Cloning: Advances and Applications

\section{Publish your work in this journal}

Stem Cells and Cloning: Advances and Applications is an international, peer-reviewed, open access journal. Areas of interest in stem cell research include: Embryonic stem cells; Adult stem cells; Blastocysts; Cordblood stem cells; Stem cell transformation and culture; Therapeutic cloning; Umbilical cord blood and bone marrow cells; Laboratory,

\section{Dovepress}

animal and human therapeutic studies; Philosophical and ethical issues related to stem cell research. This journal is indexed on CAS. The manuscript management system is completely online and includes a quick and fair peer-review system. Visit http://www.dovepress.com/ testimonials.php to read real quotes from published authors. 